\title{
The study of the perturbation of normal stresses in the rod of the I-section in the zone of application of the load
}

\author{
Sergey Saiyan and Alexander Paushkin*
}

Moscow State University of Civil Engineering, Yaroslavskoe shosse, 26, Moscow, 129337, Russia

\begin{abstract}
The study of the implementation of the Saint-Venant principle for restrained rods of the I-section exposed to various loads at its free end is carried out. When using the program complex LIRA SAPR are defined zones of disturbance of normal stresses.
\end{abstract}

\section{Research}

In previously published works $[1,2]$ authors investigated the applicability of the SaintVenant principle for rods of the rectangular cross-section. It was found that the attenuation of stress perturbations, both normal and tangential, occurs approximately at a distance of one transverse dimension. In this work, the task was to investigate the behavior of normal stresses in local areas near the loads applied at the free end of the rigidly clamped rod of the I-section (Figure 1) length of $4.8 \mathrm{~m}$. Finite element mesh $5 \times 5 \mathrm{~cm}$ was used in calculations.

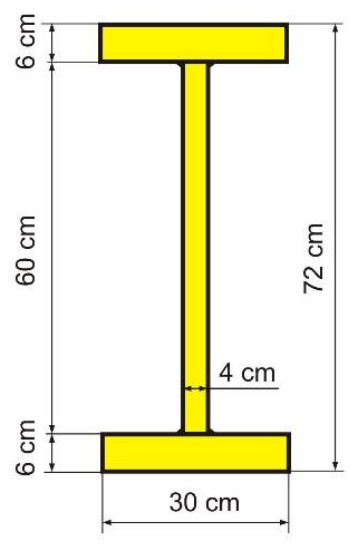

Fig. 1. Cross-section of the rod

In calculation No. 1 , a vertical force $F=1000 \mathrm{kN}$ was applied to the free end of the rod (Figure 2).

\footnotetext{
* Corresponding author: Alexglebov@bk.ru
} 

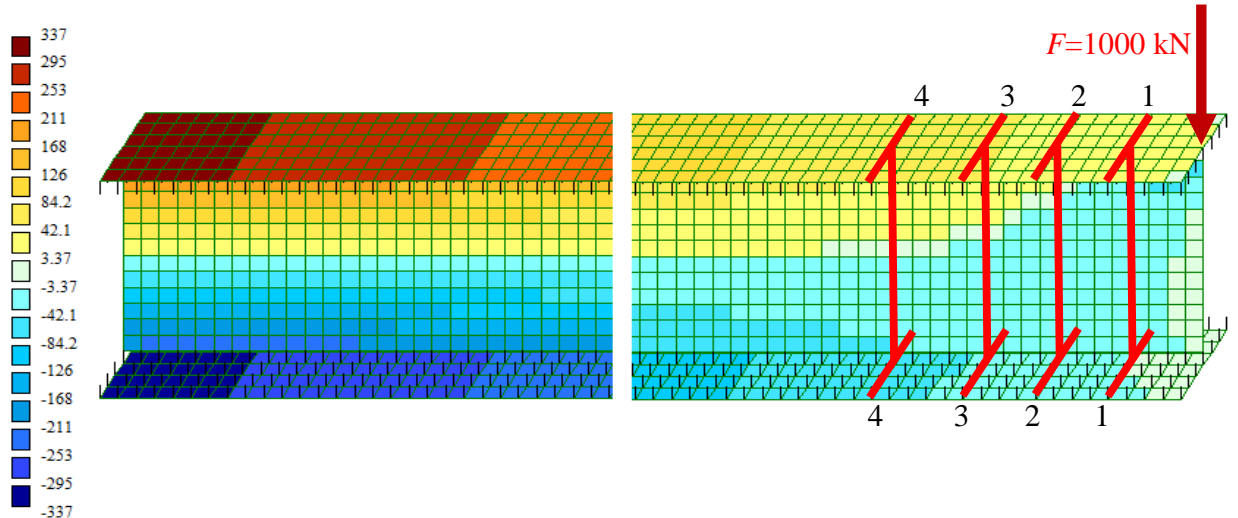

Fig. 2. Normal stresses field (calculation No. 1)

Figure 3 shows the diagrams of normal stresses for the calculation of No. 1. The sections are taken at a distance of 20,40,60 and $85 \mathrm{~cm}$ from the free end of the beam.

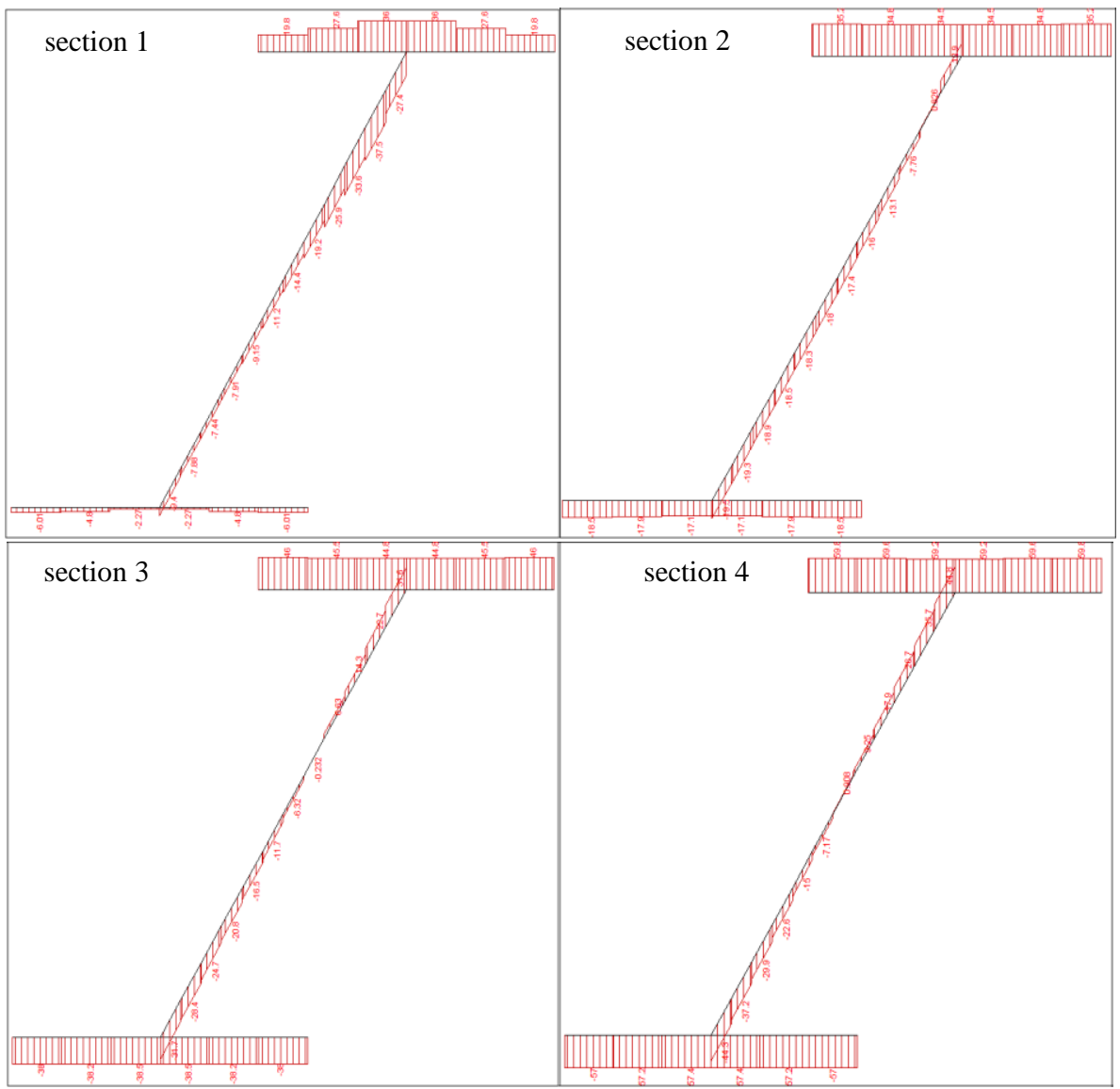

Fig. 3. Normal stress distribution diagram in sections 1 - 4 (calculation No. 1)

The criterion of convergence was the coincidence of the numerical solution with the analytical solution of the strength of materials was a discrepancy of $10 \%$. The observed 
disturbance zone was $1.1 b$ for this calculation, where $b$ is the cross-sectional vertical dimension.

In spite of the fact that the I-beam is a single section represented by three rectangular sheets connected together, the disturbance of the stressed state can be considered separately for each element. The stressed state of the web and the bottom shelf of the I-beam (the most remote place from the point of application of the load in the cross section) was considered in this calculation.

Numerical experiments have shown that the attenuation of the stress state disturbance for a web practically coincides with the results obtained for a rod of rectangular cross section [1]. For the bottom flange, we have the results shown in Figure 4.

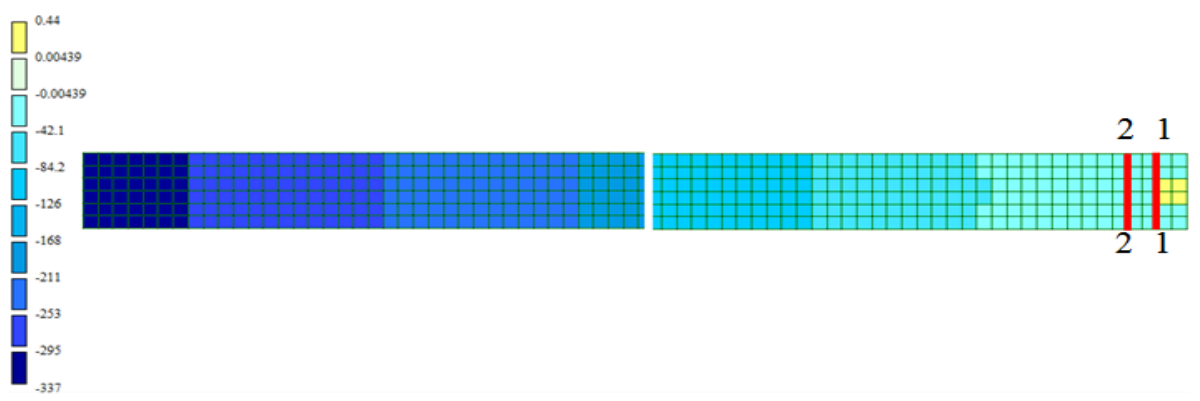

Fig. 4. Normal stresses field on the bottom flange (calculation No. 1)

Let us consider in more detail the normal stress distribution diagrams in sections of the bottom flange taken at distances of 10 and $30 \mathrm{~cm}$ (Figure 5).

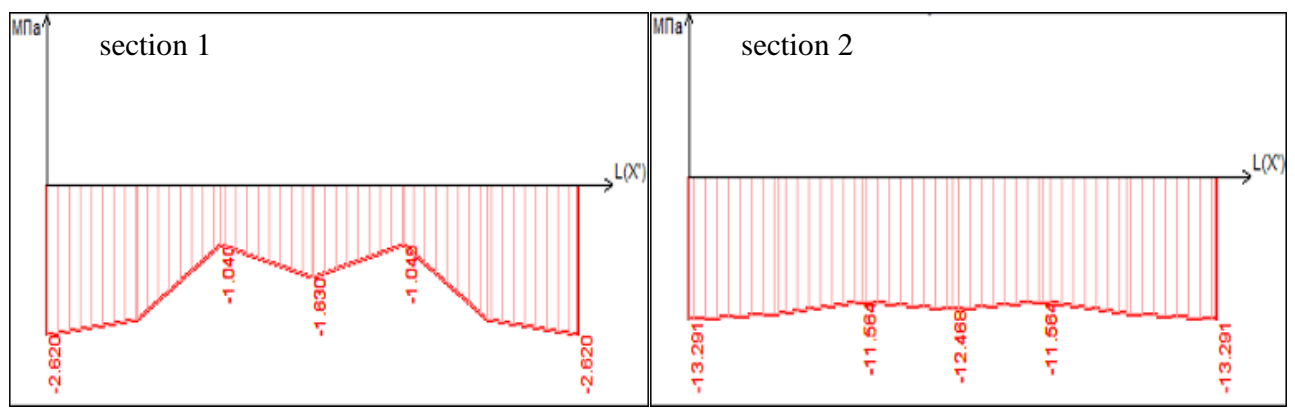

Fig. 5. Normal stress distribution diagrams in two sections of the bottom flange (calculation No. 1)

As can be seen from the diagrams of normal stresses, in the region of the interface between the web and the flange, the diagram does not have a constant character, but forms a local region of stress heterogeneity along the width of the flange, which decays along the entire length of the flange.

In the calculation of No. 2 a, the rod was loaded with a centrally applied tensile force $F=1000 \mathrm{kN}$. Figure 6 shows the normal stress field for this loading case. 


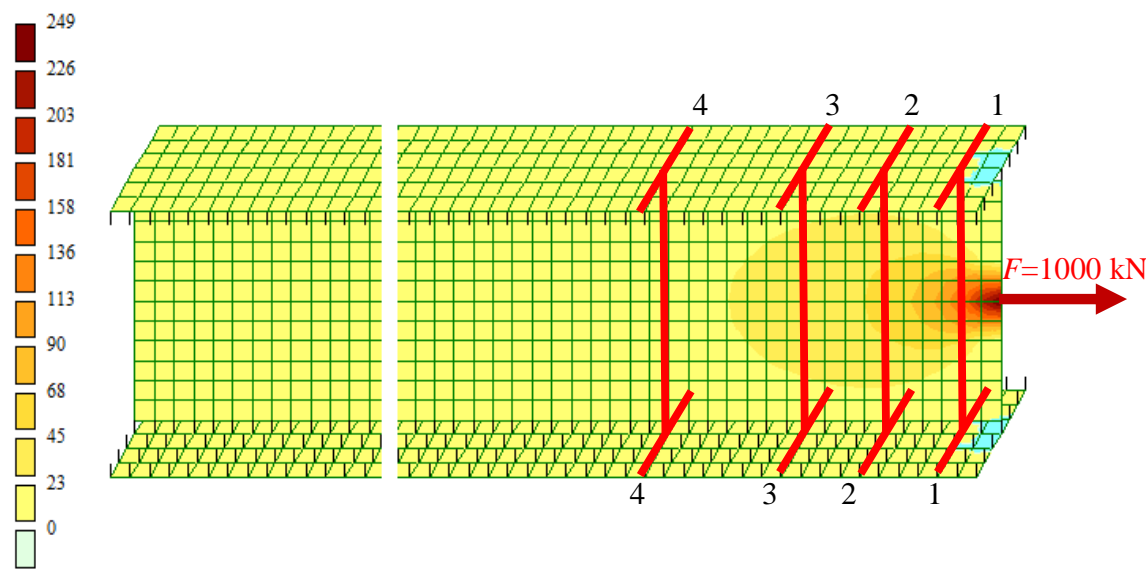

Fig. 6. Normal stresses field (calculation No. 2a)

Figure 7 shows the stress field on the top flange for this case.
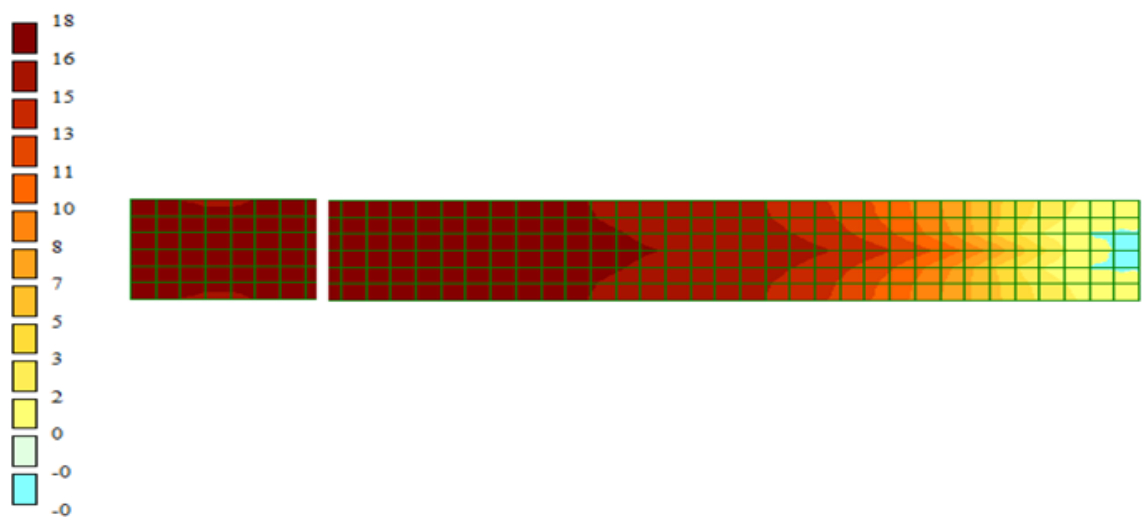

Fig. 7. Normal stresses field on the top flange (calculating No. 2a)

Based on numerical experiments to verify the applicability of the Saint-Venant principle, it was found that, for a given type of loading, the damping of the disturbance of normal stresses occurs at a distance of more than one transverse dimension of the rod. Considering the stress state of the elements separately, it can be seen that a similar phenomenon is observed both in the flange and in the web plate of the rod. Based on the results of the previous article [1], for centrally stretched rods of rectangular cross section, the damping of the perturbation of normal stresses occurred at a distance of the order of $0.92 b$, where $b$ is the transverse dimension of the rod.

Figure 8 shows the diagrams of normal stresses for calculating No. 2a in characteristic cross-sections. As can be seen from the diagrams of normal stresses along the sections (Figure 8), the damping of the normal stress disturbance occurs at a distance of $85 \mathrm{~cm}$ from the free end, which corresponds, approximately, to $1.2 b$, where $b$ is the transverse dimension of the rod. 


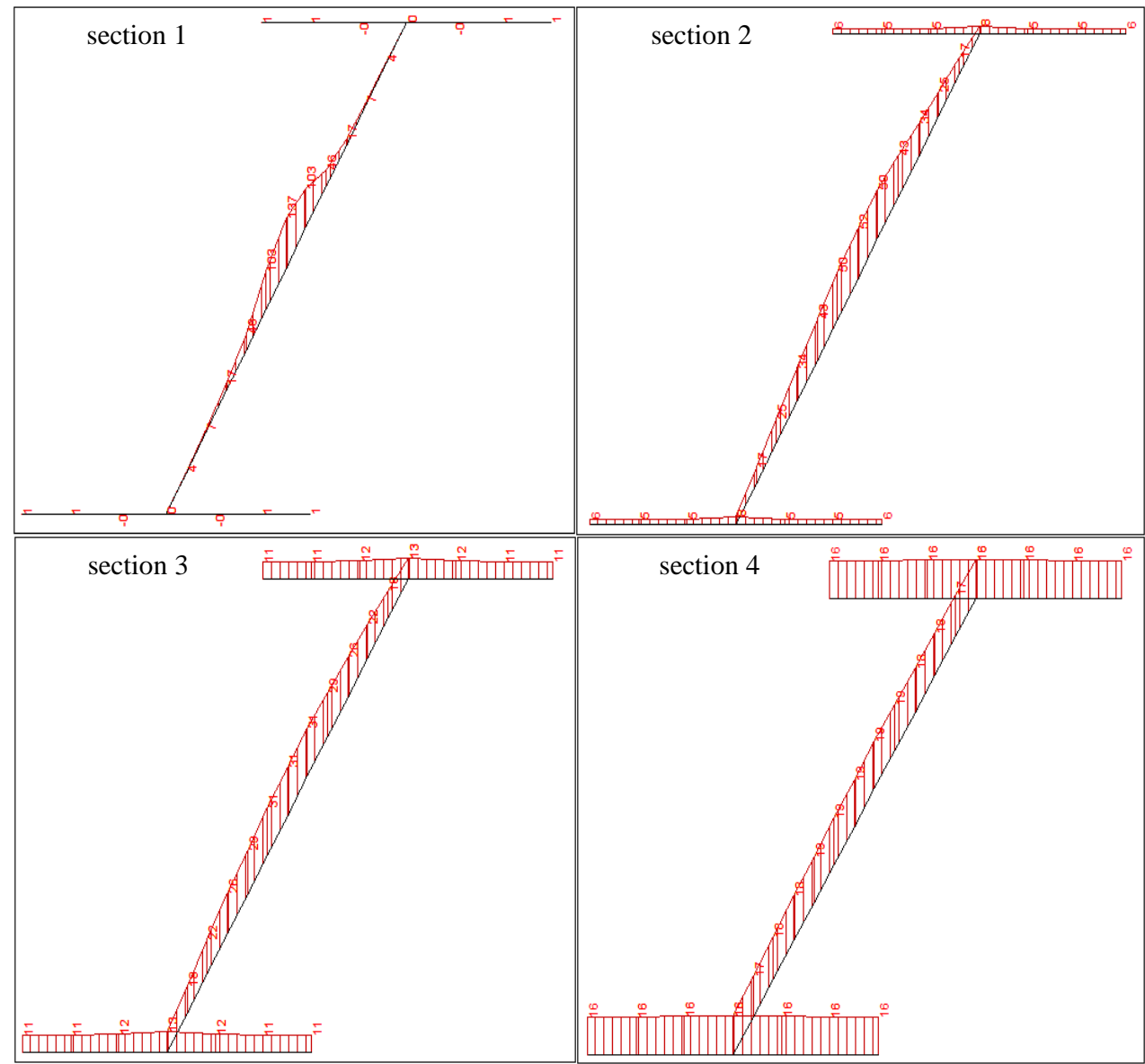

Fig. 8. Normal stress distribution diagrams in sections 1 - 4 (calculation No 2a)

However, if you apply a pair tensile load $F / 2=500 \mathrm{kN}$ (calculation No $2 \mathrm{~b}$ ) along the edges of the free end (Figure 9), then the disturbance of the stress state quickly decays at a distance of approximately $0.5 b$ from the load application area.

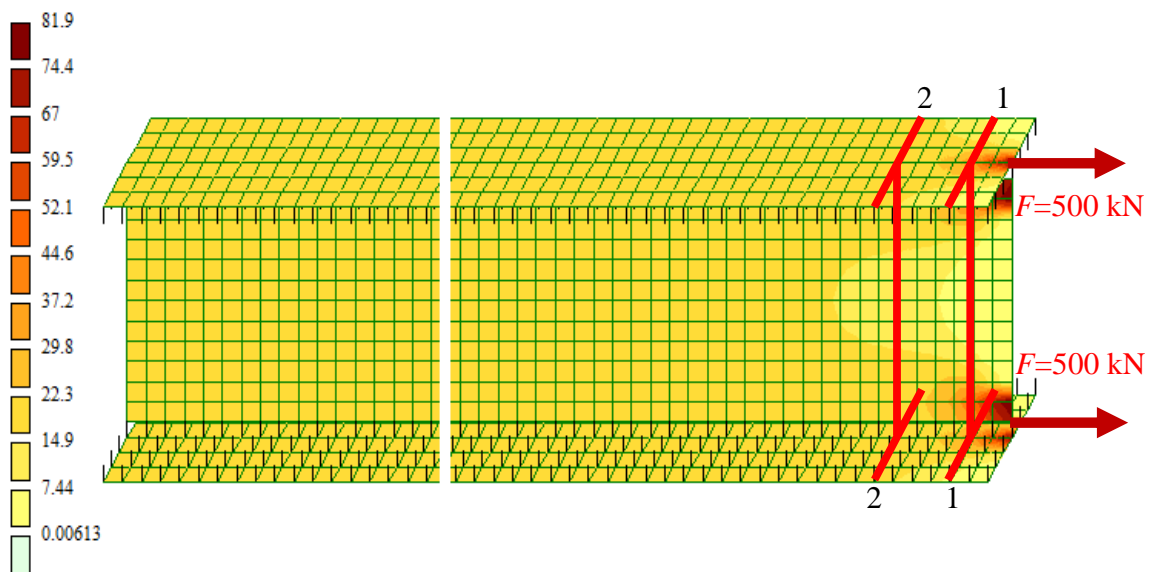

Fig. 9. Normal stresses field (calculation No 2b) 
For comparison of the attenuation characteristics of the stress state in the calculations No. 2a and No. 2b, we represent the diagrams of normal stresses for the case No.2b in two cross-sections (Figure 10) taken at the same distances from the free end as in No.2a.

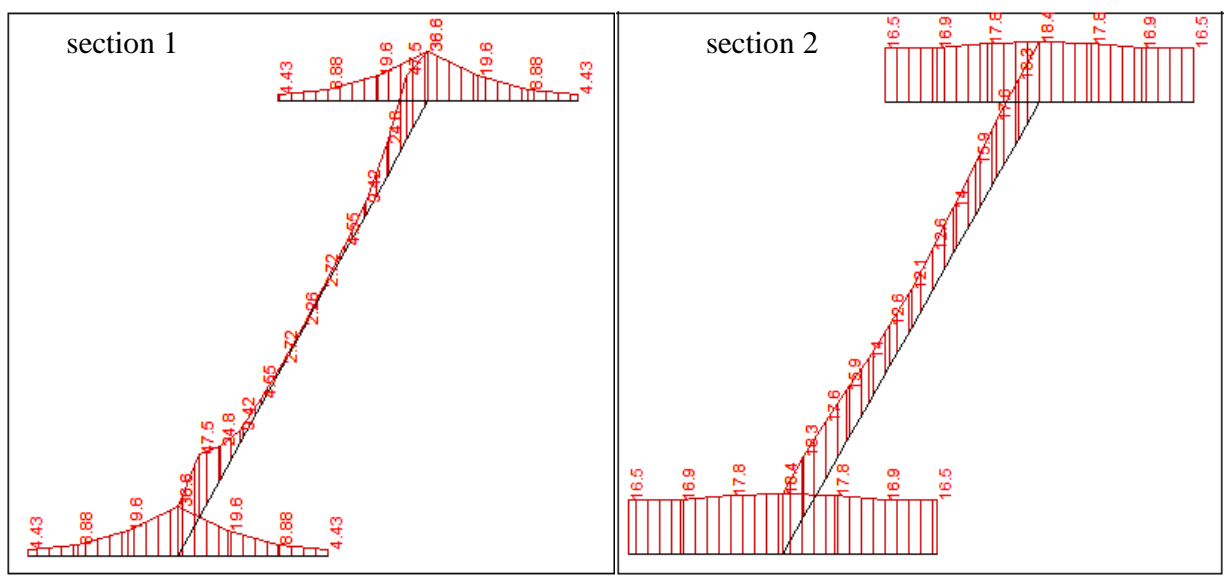

Fig. 10. Normal stress distribution diagrams in sections 1 - 2 (calculation No. 2b).

As can be seen from the diagrams of normal stresses, damping occurs faster than in case No. $2 \mathrm{a}$, at a distance of about $0.5 b$ from the free end.

The perturbation of normal stresses in the web decays at a farther distance than in the flange. Consequently, we can conclude that redistribution of stresses is most effective if the load is applied to the flanges of the I-beam, thus causing a decrease in the zone of disturbance of the stress-strain state along the length of the rod. In the case of a rectangular cross section [1], the difference in the damping zones of a stressed state under the action of a concentrated force or two forces is small $(0.95 b$ for lumped and $0.92 b$ for a pair, respectively).

This fact is confirmed by the calculation of No.3 (Figure 11), where the rod is influenced by an eccentrically applied force $F=1000 \mathrm{kN}$ at the free end.

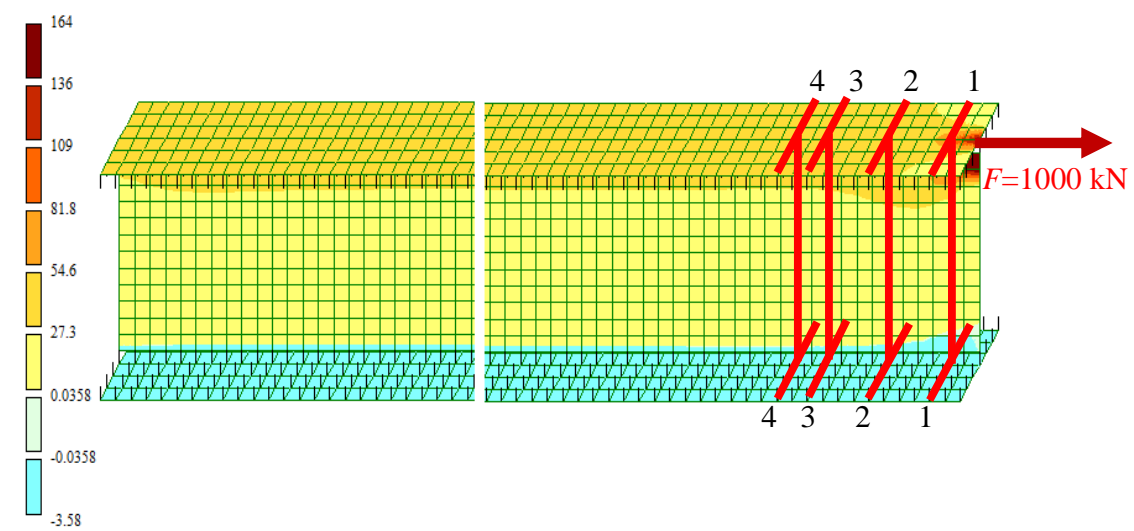

Fig. 11. Normal stresses field (calculation No. 3)

The calculation results (Figure 12) showed that the stress state perturbation in the top flange and the web plate faded at a distance of $30 \mathrm{~cm}$ from the load application zone, which corresponds to the value of $0.4 b$, and in the lower flange at a distance of $60 \mathrm{~cm}(0.8 b)$. 


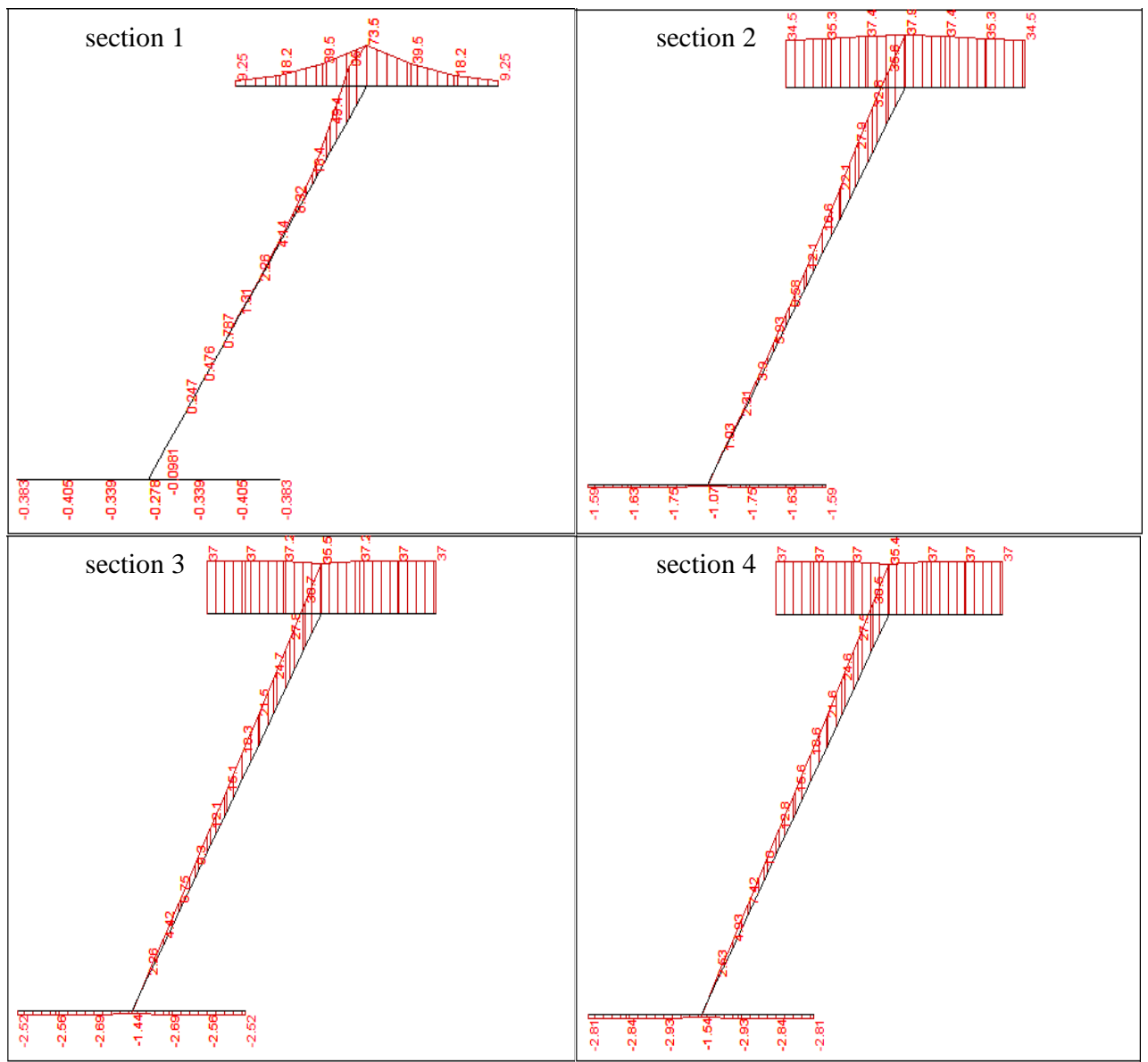

Fig. 12. Normal stress distribution diagrams in sections 1 - 4 (calculation No. 3)

We also studied the following types of beam loading.

- A pair of horizontal forces applied at close range $(10 \mathrm{~cm})$ at the end of the rod.

- Two vertical forces at the free end.

- The vertical force at the free end at the center of gravity of the I-beam

Calculations showed that the best convergence with the analytical solution is the last calculation, since, obviously, it corresponds most closely to the analytical representation of beam bending in the rod setting, where the load is applied to the axis of the rod. The attenuation of the perturbation with an allowable error of 5\% occurred already at a distance of $25 \mathrm{~cm}$ from the force application zone, which corresponds to $0.3 b$, where $b$ is the crosssectional dimension.

\section{Conclusions}

1. The linear distribution of normal stresses on the axis of symmetry in the crosssectional height for the stepped profile, adopted in the strength of materials, is generally valid for places remote from the load application zone.

2. For some types of loading, the damping of the perturbation of normal stresses was performed at a distance of $1.2 b$ (where $b$ is the transverse dimension of the rod). 
3. When considering the components of a welded I-beam, it was established that the damping of the perturbations of normal stresses in the web occurs practically in the same analogy as for a beam of rectangular cross section.

4. The attenuation of the disturbance of the stressed state in the flange occurs at a greater distance than in the web.

5. The attenuation of the stress state disturbance for a symmetric and skew-symmetric loading with respect to the neutral axis of the beam occurred over a shorter interval than in the case of an asymmetric loading scheme.

6. In general, the calculations confirmed the validity of the statement about the rapid damping of the disturbance of the stressed state in the zones remote from the site of loading and the validity of the Saint-Venant principle.

\section{References}

1. A. Paushkin, S. Saiyan, Theoretical Foundation of Civil Engineering 11 (MATEC Web of Conf., 2017)

2. A. Paushkin, S. Saiyan, Materials Science and Engineering 365 (IOP Conf. Series, 2018) 